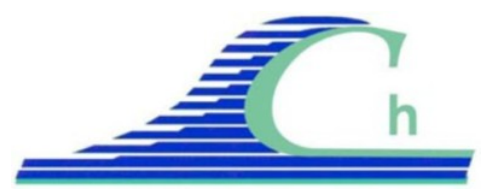

XII ${ }^{\text {ìmes }}$ Journées Nationales Génie Côtier - Génie Civil

Cherbourg, 12-14 juin 2012

DOI:10.5150/jngcgc.2012.004-D C Editions Paralia CFL

disponible en ligne - http://www.paralia.fr - available online

\title{
Hydrodynamique côtière : modélisation des courants de marée au SHOM
}

\author{
Stéphanie DESMARE ${ }^{1}$, Amandine NICOLLE ${ }^{2}$
}

1. Service Hydrographique et Océanographique de la Marine, Cellule Hydrodynamique Côtière (HDC) - Courants, SHOM/DOPS/MIP/PEP/HDC, 13, rue Chatellier, CS92803, 29228 Brest, France. stephanie.desmare@shom.fr

2. Station Biologique de Roscoff, UMR 7144 CNRS UPMC Adaptation et Diversité en Milieu Marin, Roscoff, France. amandine.nicolle@sb-roscoff.fr

\section{Résumé :}

Le SHOM, service hydrographique national, met à disposition des usagers de la mer des informations relatives aux courants marins, en particulier, des atlas de courant de marée. Ces ouvrages couvrent les côtes de France métropolitaine, Manche et Atlantique. Ils cartographient la direction et l'intensité des courants de marée à chaque "heure de marée" par rapport à un port de référence.

La modélisation des courants de marée permet d'obtenir des informations denses et une meilleure description des processus côtiers. Cet article présente une partie de l'activité du SHOM en hydrodynamique côtière : d'une part les modèles 2D, à l'origine des atlas de courant de marée qui représentent, à la précision des mesures près, les champs instantanés des courants de marée barotrope. Les modèles 3D, d'autre part, qui apportent des informations supplémentaires sur la structure verticale du courant. La dernière partie présente les applications à partir des modèles 3D développés en Manche, notamment, un exemple de nouveau produit répondant à de nombreux besoins.

Mots-clés : Génie côtier - Hydrodynamique côtière - Marées - Courants Modélisations - Approches statistiques

\section{Abstract:}

The SHOM, French national hydrographic service, provides to sea users tidal stream information in tidal stream charts. These documents cover the Atlantic and English Channel French coast.

The atlases present a comprehensive view of flow speeds and bearings at hourly intervals relative to high water at some reference harbor.

Modeling tide and tidal currents allow obtaining more information and a better description of coastal processing. This paper presents a part of the coastal hydrodynamic studies done at SHOM: first 2D models used to provide tidal stream charts are able to describe barotropic tide flow with a sufficient accuracy. 3D models provide more information on the vertical flow profile. The last part describes some applications in use at SHOM, from the 3D model set up in the English Channel to new products that respond to sea user's needs. 


\section{Introduction}

Dans le domaine maritime, la connaissance et la prévision des courants sont nécessaires pour répondre aux besoins d'informations nautiques et appréhender leurs impacts sur la sécurité de la navigation, sur les opérations (en surface et sous-marines) et autres activités maritimes. Le système de prévision des courants de marée développé par le SHOM a été éprouvé sur les zones de France métropolitaine. Des modèles numériques ont ainsi été mis en œuvre à l'aide du code de calcul TELEMAC-2D et maintenant TELEMAC-3D développé par le Laboratoire d'Hydraulique Saint-Venant (http://www.opentelemac.org/).

Neufs modèles en deux dimensions ont été développés le long des côtes de France métropolitaine et ont servi pour élaborer les atlas régionaux de courant diffusés par le SHOM (voir §5.1). Depuis 2009, 5 modèles en trois dimensions ont été développés (à différentes échelles spatiales) en Manche et Atlantique pour satisfaire de nouveaux besoins en calculant les courants sur toute la colonne d'eau.

Ces modèles permettent de décrire les courants de marée d'une zone marine avec des résolutions spatiales relativement fines (100 km au large, quelques dizaines de mètres à la côte) et avec une précision suffisante pour les besoins civils et militaires. Les résultats numériques obtenus montrent un bon accord avec les hauteurs d'eau et les courants calculés à partir des observations faites in situ.

\section{Méthode de calcul}

\subsection{Objectifs de la modélisation au SHOM}

Le SHOM, dispose aujourd'hui d'outils de modélisation numérique qui sont utilisés pour diverses applications intéressant particulièrement l'hydrographie notamment pour la réduction des sondages : la simulation de la marée sur une longue période, suivie du calcul des constantes harmoniques, permet d'obtenir une marée théorique en tout point du maillage (voir figure 1). Ces types de résultats sont exploités par la suite pour le calcul des conditions limites des modèles hydrodynamiques et les prédictions de marée. La production des atlas de courant de marée utiles aux navigateurs est issue de la modélisation des courants de marée (vitesse et direction) en zone côtière.

Le développement et le maintien des systèmes d'analyse, de prédiction et de modélisation de la marée et des courants sont nécessaires pour avoir une amélioration continue de la connaissance de la marée. Il s'agit alors d'étudier les principaux processus agissant sur la modélisation hydrodynamique (frottement sur le fond, bathymétrie, turbulence verticale) et d'améliorer la précision pour la prédiction des courants et des hauteurs d'eau. 


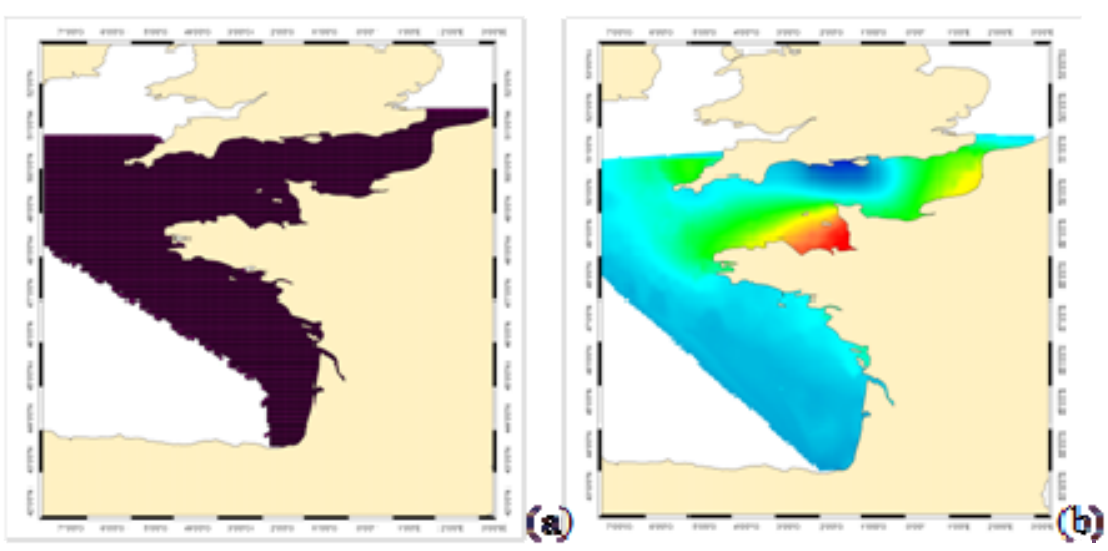

Figure 1. Modèle de marée CSTFRANCE (a)Emprise sur les côtes métropolitaines, (b) Amplitude M2. (SHOM).

\subsection{Logiciel hydrodynamique}

Depuis 1996, les modèles de marée mis en place au SHOM sont calculés à l'aide du code de calcul TELEMAC. Différents modules permettent de modéliser la dynamique des écoulements, bi ou tridimensionnels, dans les milieux côtiers et le transport de substances par ces écoulements : polluants conservatifs ou dégradables, sables, vases, etc.

Les algorithmes performants basés sur la méthode des éléments finis permettent une discrétisation de l'espace sous forme de maillage à éléments triangulaires, ce qui permet de raffiner le maillage dans les zones présentant un intérêt particulier (notamment pour la navigation : ports, chenaux). Cela permet également une modélisation fine des processus physiques côtiers ou encore de prendre en compte une configuration topographique particulière ou la complexité du trait de côte.

Le code de calcul bidimensionnel TELEMAC-2D permet de simuler les écoulements à surface libre à deux dimensions. En résolvant les équations de Saint-Venant, le logiciel calcule, en chaque point du maillage, la hauteur d'eau ainsi que les composantes du courant (vitesses moyennées sur la verticale).

En 3D, TELEMAC-3D résout les équations de Navier-Stokes pour des échelles horizontales allant de quelques kilomètres (modèle Manche, voir figure 2a) à une trentaine de mètres (modèle de la Baie de Somme, voir figure 2b). Il fournit pour chaque nœud, la hauteur ainsi que les composantes de la vitesse.

\section{Construction d'un modèle}

\subsection{Données d'entrée}

Les données nécessaires à la mise en place du modèle sont :

- les données bathymétriques (MNT de sondes brutes, cartes marines, BDBS -Base de données bathymétrique du SHOM-) ; 
- le trait de côte (mondial, Histolitt) ;

- les mesures de marée (hauteur d'eau, niveau moyen);

- les données d'observation de courant au point fixe.

Généralement ces données doivent faire l'objet de traitements particuliers pour pouvoir être utilisées dans le modèle (NICOLLE \& PINEAU, 2009). Une adaptation régionale doit être faite pour certains paramètres du modèle liés au choix des caractéristiques physiques propres à la zone d'étude (densités de l'eau de mer, type de fond pour le coefficient de frottement, coefficient de diffusion des vitesses, viscosité au niveau des parois, etc.) ainsi que pour le calcul des conditions aux limites (hauteur d'eau, vitesses de courant, apports fluviaux, etc.).

\subsection{Résolution spatiale}

Les différents modèles ont été mis en place le long des côtes de France métropolitaine à des échelles plus ou moins grandes en fonction des informations et des raffinements souhaités notamment au niveau des côtes et des infrastructures portuaires (voir figure $2 \mathrm{~b}$ ). La résolution verticale est de quelques mètres par petits fonds (de l'ordre de 10 à 20 couches). Le choix des dimensions des mailles est primordial puisque plus celle-ci est petite, plus la résolution et donc la précision des résultats sont bonnes. Cependant, un compromis semble nécessaire car il faut tenir compte de l'emprise du modèle, du nombre de mailles à utiliser et du pas de temps choisi. Tous ces choix influent sur la stabilité numérique du modèle et les temps de calcul engendrés.

La connaissance de la bathymétrie est un autre critère de dimensionnement des mailles puisque la résolution du modèle ne peut se faire qu'à une échelle spatiale équivalente à la densité de points disponibles.

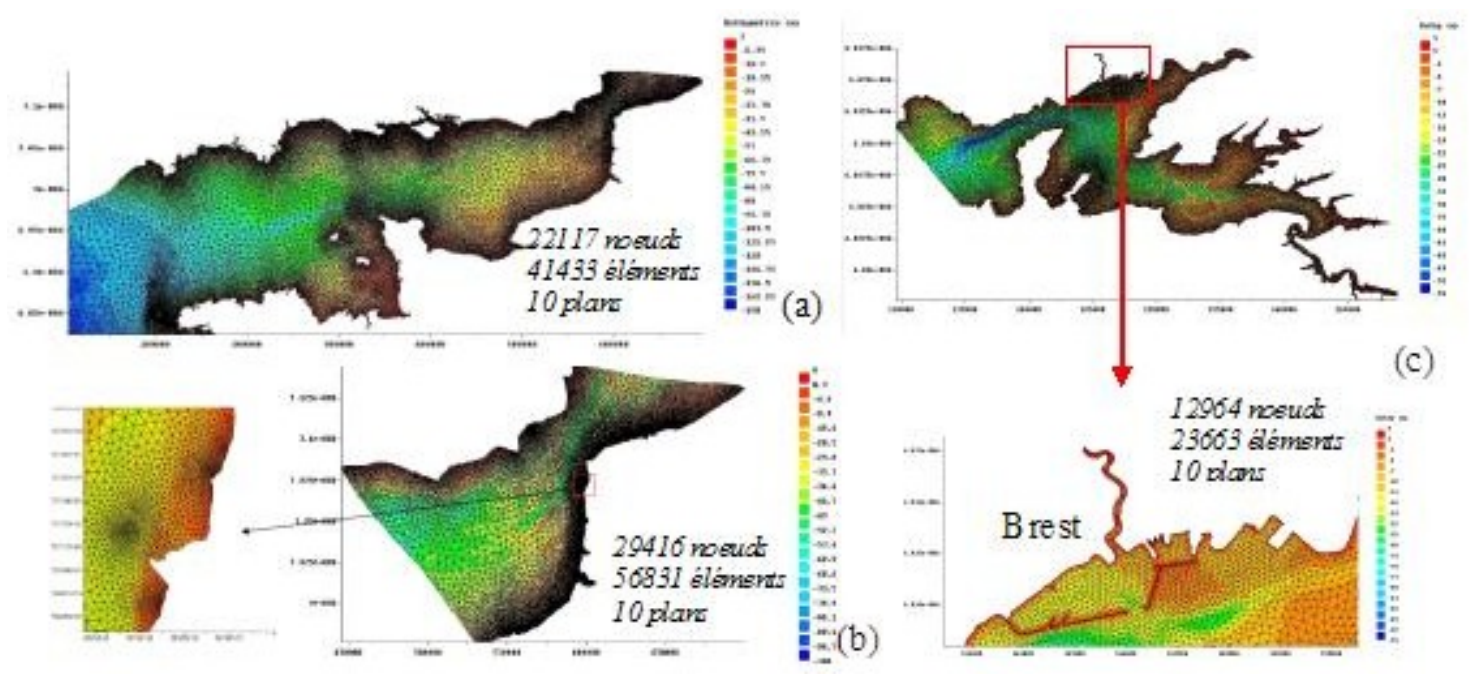

Figure 2. Maillage triangulaire - bathymétrie et résolutions des modèles $3 D(a)$ Manche : $100 \mathrm{~m}$ à $20 \mathrm{~km}$ au large (b) Baie de Somme-Pas de Calais : $13 \mathrm{~m}$ à $6 \mathrm{~km}$ au large, (c) Rade de Brest : $10 \mathrm{~m}$ à la côte à $500 \mathrm{~m}$ au large. 


\section{XII ${ }^{\text {èmes }}$ Journées Nationales Génie Côtier - Génie Civil \\ Cherbourg, 12-14 juin 2012}

\subsection{Réalisations}

Les résultats (marée et courants) sont disponibles sur les côtes métropolitaines pour deux conditions type de marée (coefficients 45 et 95). Le coefficient de frottement sur le fond est pris en compte et calculé à partir de la loi de Chézy (avec un coefficient constant ou variable choisi en fonction de la nature des fonds).

Pour l'application marée au SHOM, le modèle ne reproduit que les courants de marée. Les forçages atmosphériques (pression, vent, chaleur et précipitations) ne sont pas pris en compte.

La surface libre est imposée aux frontières ouvertes soit à partir des données extraites du modèle de marée (CSTFRANCE, voir figure 1), soit à partir des observations disponibles (voir figure 3a). Dans les deux cas, ces données sont interpolées spatialement pour être imposées en chaque point de la frontière et temporellement pour être imposées à chaque pas de temps du calcul. Ce mouvement forcé se propage d'une maille à l'autre dans tout le domaine de calcul (SIMON, 2007, chapitre 6).

Enfin, selon les régions où l'influence des estuaires ou rivières n'est pas négligeable, un débit moyen est imposé au niveau des frontières liquides.

\section{Résultats}

La validation des modèles est effectuée par comparaison des résultats de la simulation avec des hauteurs d'eau prédites à partir d'observations (CREACH \& TEXIER, 2011) et de mesures de courants enregistrées à plusieurs immersions en différentes stations (voir figure $3 b)$.
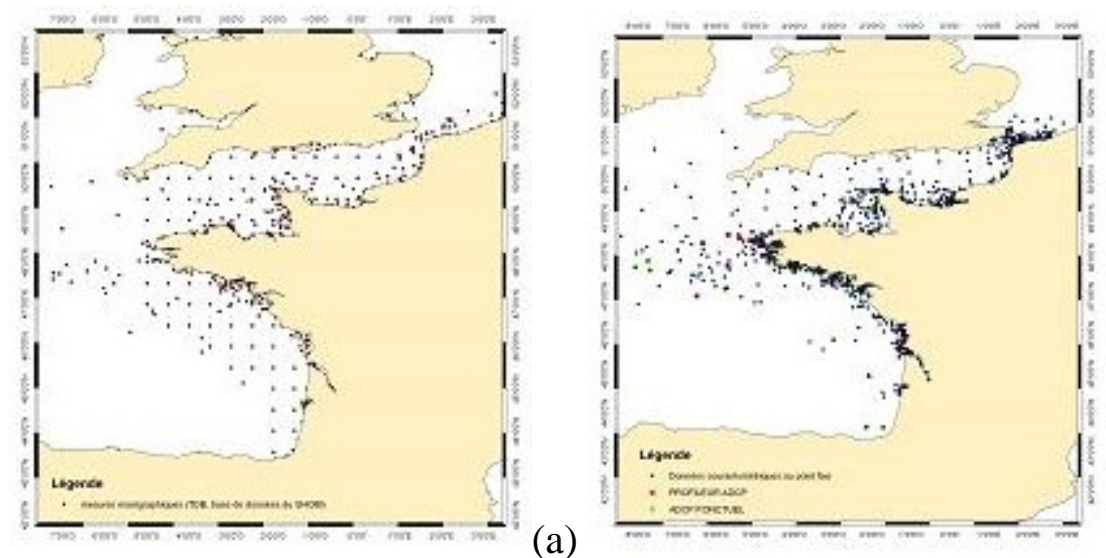

(b)

Figure 3. Position des points de mesures (a) de hauteur d'eau (TDB, SHOM), (b) de courant - en France Métropolitaine contenues dans les bases de données du SHOM.

Pour la qualification des modèles, les comparaisons portent alors sur les hauteurs d'eau en calculant d'une part les écarts en amplitude (pour la pleine mer) des hauteurs d'eau prédites aux différents points de mesures existants (voir figure 3a) et les hauteurs 
extraites du modèle en ces mêmes points ; et d'autre part les déphasages moyens en heure entre les phases des pleine mer et basse mer théoriques et les phases de pleine mer et basse mer observées.

La validation des résultats obtenus pour les courants est réalisée en calculant la moyenne des écarts entre les amplitudes en flot et jusant des vitesses théoriques (calculées par le modèle) et les vitesses obtenues aux points de mesures (voir figure 3b) ainsi que le déphasage moyen en heure entre les phases des courants des pleine mer et basse mer théoriques et les phases des courants mesurés.

Les tableaux ci après résument les résultats obtenus pour les modèles $3 \mathrm{D}$ et illustrent les apports par rapport au 2D, issu des précédentes réalisations.

Tableau 1. Comparaisons et précisions des modèles de courants de marée pour les hauteurs d'eau pour un coefficient de marée de 95 (NICOLLE, 2009 ; 2010a ; 2010b; $2011 a ; 2011 b)$.

\begin{tabular}{|c|c|c|c|c|c|}
\hline Modèle & $\begin{array}{l}\text { Points de } \\
\text { validation }\end{array}$ & $\begin{array}{l}\text { Ecart } \\
\text { amplitude }(\mathrm{cm})\end{array}$ & écart-type (cm) & $\begin{array}{l}\text { Déphasage BM } \\
\text { (minutes) } \sigma= \\
\text { écart-type }\end{array}$ & $\begin{array}{l}\text { Déphasage PM } \\
\text { (minutes) } \sigma= \\
\text { écart-type }\end{array}$ \\
\hline Rade de Brest $3 D$ & 13 & 4.7 & 2.4 & $9(\sigma=6)$ & $3(\sigma=2)$ \\
\hline Manche 2D (1999) & 172 & 15.3 & 12.3 & $13(\sigma=13)$ & $11(\sigma=10)$ \\
\hline Manche $3 D$ & 190 & 9.1 & 7.1 & $8(\sigma=12)$ & $8(\sigma=7)$ \\
\hline Baie de Seine $3 D$ & 25 & 7.6 & 7.1 & $5(\sigma=3)$ & $6(\sigma=4)$ \\
\hline $\begin{array}{l}\text { Baie de Somme - } \\
\text { Pas de Calais } 3 D\end{array}$ & 28 & 6.4 & 4.1 & $4(\sigma=3)$ & $8(\sigma=6)$ \\
\hline Mer d'Iroise 3D & 39 & 3.6 & 3.1 & $5(\sigma=2)$ & $5(\sigma=2)$ \\
\hline
\end{tabular}

Tableau 2. Comparaisons et précisions des modèles de courants de marée pour les vitesses pour un coefficient de marée de 95 (NICOLLE, 2009 ; 2010a; 2010b; 2011a; 2011b).

\begin{tabular}{|c|c|c|c|c|c|}
\hline Modèle & $\begin{array}{l}\text { Points de } \\
\text { validation }\end{array}$ & $\begin{array}{l}\text { Ecart flot } \\
(\mathrm{cm} / \mathrm{s}) \\
\sigma=e ́ c a r t-t y p e \\
\end{array}$ & $\begin{array}{l}\text { Ecart jusant } \\
(\mathrm{cm} / \mathrm{s}) \\
\sigma=e ́ c a r t-t y p e)\end{array}$ & $\begin{array}{l}\text { Déphasage } B M \\
\text { (minutes) } \sigma= \\
\text { écart-type }\end{array}$ & $\begin{array}{l}\text { Déphasage PM } \\
\text { (minutes) } \sigma= \\
\text { écart-type }\end{array}$ \\
\hline Rade & $21(4$ & $14.4(\sigma=8.9)$ & $12.6(\sigma=9.4)$ & $7(\sigma=7)$ & $16(\sigma=14)$ \\
\hline Manche 2D & 121 & $19.9(\sigma=21.2)$ & $23.3(\sigma=23.2)$ & $24(\sigma=23)$ & $33(\sigma=32)$ \\
\hline Manche $3 D$ & $\begin{array}{l}173(25 \\
A D C P)\end{array}$ & $15.3(\sigma=15.2)$ & $17.1(\sigma=15.3)$ & $21(\sigma=25)$ & $20(\sigma=20)$ \\
\hline Baie de Seine 2D & & $46.9(\sigma=47.2)$ & $40.4(\sigma=42.2)$ & $36(\sigma=32)$ & $39(\sigma=32)$ \\
\hline Baie de Seine 3D & $18(3 A D C P)$ & $14.4(\sigma=15.7)$ & $11.2(\sigma=12.3)$ & $13(\sigma=9)$ & $12(\sigma=7)$ \\
\hline $\begin{array}{l}\text { Baie de Somme - } \\
\text { Pas de Calais } 3 D\end{array}$ & $28(1 A D C P)$ & $15.5(\sigma=13.9)$ & $13.4(\sigma=13.3)$ & $11(\sigma=7)$ & $12(\sigma=97)$ \\
\hline Mer d'Iroise 3D & $52(8 A D C P)$ & $14.9(\sigma=12.1)$ & $12.9(\sigma=11.4)$ & $6(\sigma=7)$ & $6(\sigma=7)$ \\
\hline
\end{tabular}




\section{XII ${ }^{\text {èmes }}$ Journées Nationales Génie Côtier - Génie Civil \\ Cherbourg, 12-14 juin 2012}

La figure 4 ci-après montre un exemple des comparaisons (Résultat des modèles 2D et 3D versus mesures) faite pour le modèle Manche pour un coefficient de marée de 95 (NICOLLE, 2010 a).
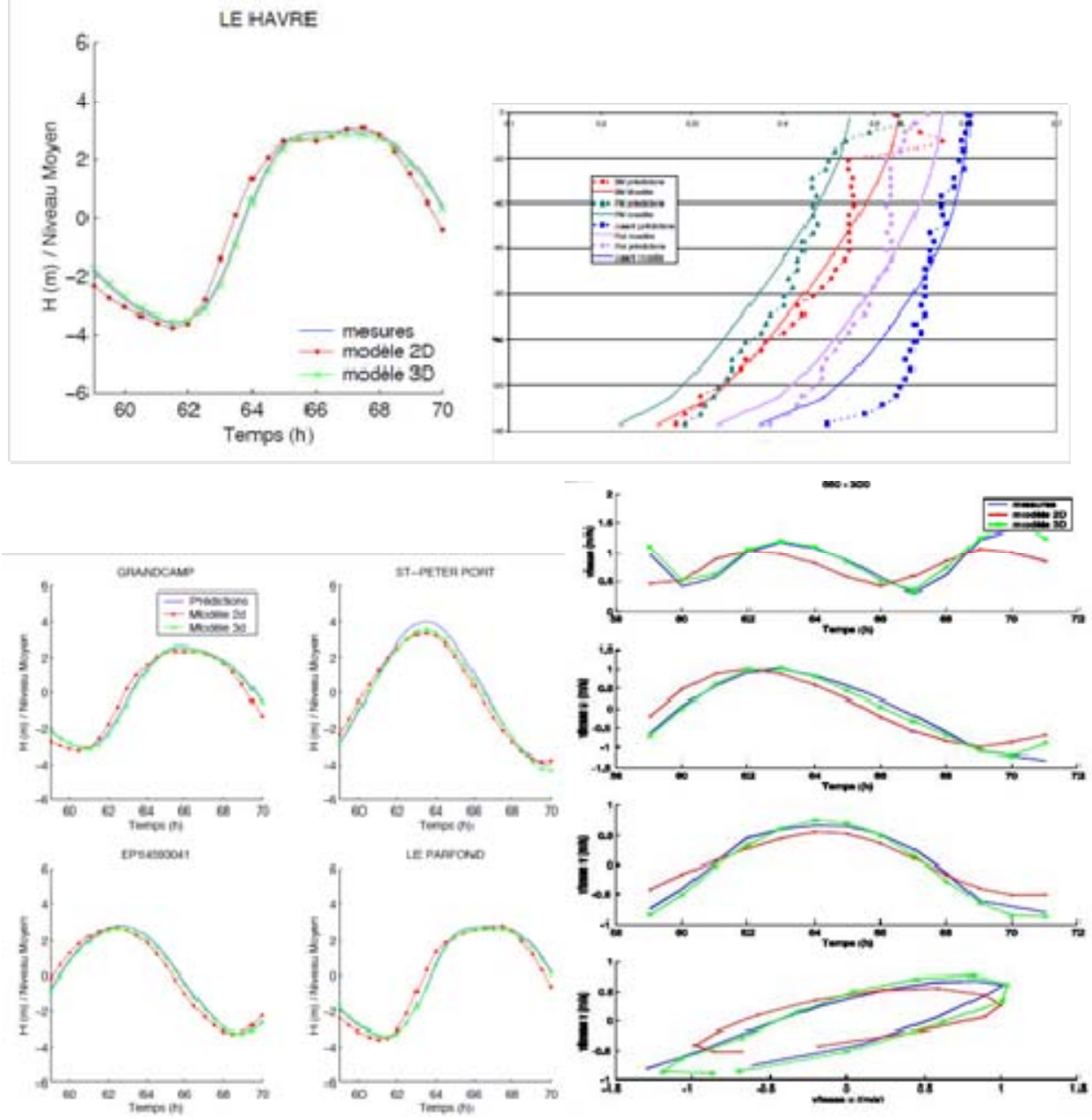

Figure 4. Validation du modèle 3D Manche. (NICOLLE, 2010 a).

L'évolution des modèles numériques et le passage au 3D s'est traduite par une amélioration des prédictions (augmentation de la précision des résultats) liée à :

- une meilleure résolution spatiale au niveau côtier et accès aux ports ;

- une meilleure prise en compte des processus importants (bathymétrie, frottement sur le fond, turbulence) ;

- une meilleure connaissance des zones étudiées (densité de mesures in situ);

- une meilleure représentation des cisaillements ;

- les performances accrues du code TELEMAC depuis son début d'utilisation au SHOM (EDF, 1997 ; EDF, 2007). 


\section{Exploitation des modèles}

\subsection{Production des atlas de courant}

Les modèles validés par comparaison aux mesures (hauteurs d'eau et vitesses) sont à la base des atlas de courants publiés par le SHOM. Ces ouvrages cartographient la direction et l'intensité des courants de marée à chaque heure "marée" par rapport à un port de référence.
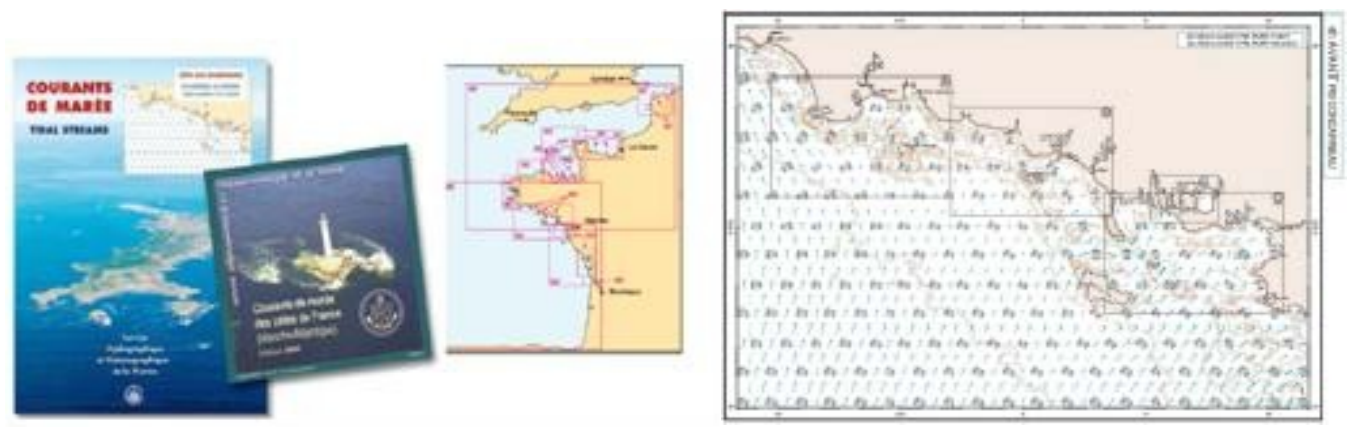

Figure 5. Exemple d'exploitation des modèles de courant 2D.

\subsection{Nouveaux produits}

L'élaboration des modèles 3D de courants de marée et l'évolution des besoins (défense et civils : EMR, aquaculture, hydrolien marin, etc.) conduisent à définir de nouveaux produits susceptibles de fournir des informations pertinentes sur les courants de marée, en qualifiant les courants de surface mais également de fond et à des immersions données (voir figure 6).
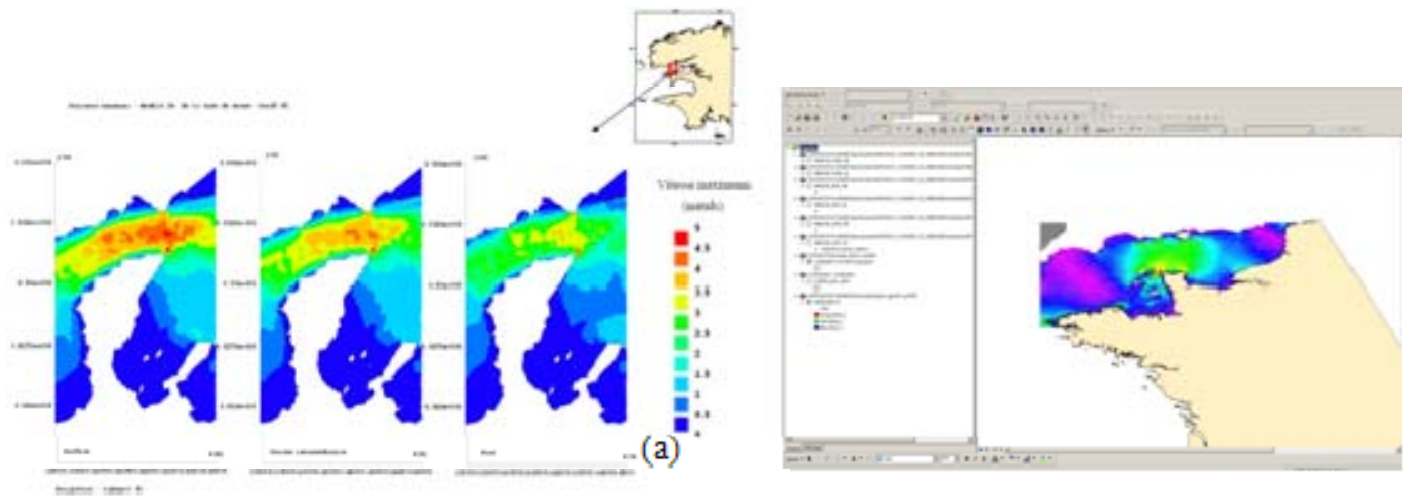

(b)

Figure 6. Exemple de visualisation des résultats : (a) Extrait du modèle Rade de Brest $3 D$ à différentes immersions, (b) Extrait du produit Manche 3D sous ARCGIS.

Des exemples de secteurs et domaines d'applications sont la navigation de surface et sous-marine; l'optimisation des opérations et des moyens à déployer (défenses et civils). 


\section{XII ${ }^{\text {èmes }}$ Journées Nationales Génie Côtier - Génie Civil \\ Cherbourg, 12-14 juin 2012}

\section{Conclusions}

Ces différentes réalisations montrent des résultats satisfaisants et l'intérêt de l'utilisation de la modélisation numérique pour caractériser les courants de marée en zone côtière On pourra noter l'apport de la modélisation 3D pour la connaissance des profils de courant sur la colonne d'eau.

Les observations de hauteur d'eau, les mesures de courant in situ et une bonne connaissance de la bathymétrie restent indispensables pour élaborer, calibrer et valider ces modèles. Les applications possibles sont multiples et les besoins évoluent, il faut donc poursuivre l'enrichissement des bases de données et l'amélioration continue des modèles.

\section{Références bibliographiques}

CREACH R., TEXIER C. (2011). Refonte de la base des données in situ du niveau de la mer Tide DataBase (TDB). Annales Hydrographiques 777.

EDF (1997). Logiciel TELEMAC-3D Version 2.2 - Note de principe.

EDF (2007). Logiciel TELEMAC-3D Version 5.8 - Manuel de l'utilisateur.

NICOLLE A. (2009).Réalisation d'un modèle 3D de courants de marée dans la rade de Brest. Rapport d'étude SHOM n.2009-176.

NICOLLE A. (2010a). Réalisation d'un modèle 3D de courants de marée dans la Manche. Rapport d'étude SHOM n²011-044.

NICOLLE A. (2010b). Réalisation d'un modèle 3D de courants de marée dans la baie de Seine. Rapport d'étude SHOM n²011-052.

NICOLLE A. (2011a). Réalisation d'un modèle 3D de courants de marée dans la baie de Somme -Pas de calais. Rapport d'étude SHOM n²011-076.

NICOLLE A. (2011b). Réalisation d'un modèle 3D de courants de marée en mer d'Iroise. Rapport d'étude SHOM n²011-104.

NICOLLE A., PINEAU L. (2009). Modélisation numérique en hydrodynamique côtière. Guide technique SHOM n²007-050.

SIMON B. (2007). La marée océanique côtière. Coédition de l’Institut Océanographique et du SHOM, 433 p.

SHOM. Guide utilisateur : Base de données des mesures de hauteurs d'eau - Tide DataBase (TDB), 83 p. 
Thème 1 - Hydrodynamique côtière 